AperTO - Archivio Istituzionale Open Access dell'Università di Torino

\title{
Education, Dynamic Signalling and Social Distance
}

\section{This is the author's manuscript}

Original Citation:

\section{Availability:}

This version is available http://hdl.handle.net/2318/135173

since

Published version:

DOI:10.1093/oep/gpn014

Terms of use:

Open Access

Anyone can freely access the full text of works made available as "Open Access". Works made available under a Creative Commons license can be used according to the terms and conditions of said license. Use of all other works requires consent of the right holder (author or publisher) if not exempted from copyright protection by the applicable law. 


\title{
Education, dynamic signalling, and social distance
}

\author{
By Andrea Gallice
}

BRICK, Collegio Carlo Alberto, Via Real Collegio 30, 10024, Moncalieri, Italy, and University of Siena; e-mail: andrea.gallice@brick.carloalberto.org

\begin{abstract}
In many European countries the average level of education has grown steadily over the last few generations. The paper shows how such a trend can be easily rationalized by a model that extends a standard signalling game in two directions. First, a temporal structure is induced by having two different cohorts of individuals playing the game. Second, to standard rational agents, the model postulates the existence of two classes of agents that care about their relative position in the distribution of educational choices. Conformist individuals wish to be close to the average level; status seeking individuals seek to be above it. Results show that the presence of these two classes of individuals generates an average level of education that changes and increases over time for a wide range of choice of parameters. Consequences of this increasing level of education on the behaviour of the firms are also explored.
\end{abstract}

JEL classifications: I20, D82.

\section{Introduction}

Over the past few decades, the average level of education increased steadily in almost every European country. For example, Eurostat (2001) reported that in 2001, over $75 \%$ of individuals in the 25-29 year age group had completed at least their upper secondary education compared with 52\% of individuals in the 50-64 year age group. Further, Eurostat (2005) reported that the total number of graduates in the EU25 increased by more than 30\% during the period 1998-2003. The causes contributing to the shaping of such important changes are obviously manifold, including a favourable sociopolitical situation, increasing wealth, sustained unemployment rates and rapid technological progress. In addition to these explanations, this paper shows that recent educational dynamics are easily accounted for by a simple signalling model (Spence, 1973) enriched by the presence of individuals who care about their relative position in the educational distribution.

We argue that there are two kinds of reasons why people may care about their relative level of education. First, there are material reasons related to the chance of finding a suitably attractive job in the labour market. A consequence of the increase of the general level of education has been a reduction in the 'value' of a given level of schooling. For example, a bachelor's degree two generations ago was a very valuable 
asset and an effective signal to the labour market. Today, the same degree is much less informative and individuals need higher qualifications in order to differentiate themselves from other applicants.

A second set of reasons relates to the social and psychological aspects associated with the acquisition of education. The level of education is a signal that individuals send not only to firms, but also to the rest of society. In many social contacts, there is a favourable bias towards more educated individuals as people are usually willing to interpret education as a proxy for qualities, such as ability, knowledge, and persistence. In contrast, individuals with a low level of education are often judged unfavourably, no matter what their effective abilities may be. Again, this social evaluation of a given level of schooling depends on the average level of education in society. ${ }^{1}$

Therefore, an individual's choice of his level of schooling must take into account not only the absolute level of schooling acquired but more importantly his level of schooling in relation to others in his cohort. This means that individual preferences about education are interpersonally dependent, a feature which is usually neglected by standard models of educational choices. To clarify how the effectiveness of this double-signalling effect (towards the firms and towards the rest of society) is related to the average level of education, consider the example of a student who obtains a high grade on an important exam. The utility the student enjoys from such an achievement is clearly influenced by the average grade of the class. If the average grade is low, the signal the student sends to the teacher is more informative and thus, more likely to help him during his scholastic career. At the same time, the student sends a stronger signal to her colleagues. The perception of this second component depends on the student's preferences. For instance, his ego could be gratified or the student could feel some embarrassment by standing out so clearly.

This paper focuses on the choice made by an individual about the level of his education attainment by considering his innate characteristics, as well as his ambitions. It combines a standard model of signalling with theories of social distance. More precisely, the paper analyses educational dynamics in a heterogeneous population of myopic agents who have different productivity as well as different attitudes about their relative educational achievement. Some individuals, possibly the majority, are not influenced by considerations of social distance (independent individuals); some try to differentiate themselves from other agents and reach a higher status (status-seeking individuals); others adopt a more conformist behaviour (conformist individuals).

The presence of non-selfish agents modifies considerably the standard results of static signalling models. ${ }^{2}$ In particular, under very weak assumptions about the

\footnotetext{
${ }^{1}$ Individuals can be more or less sensitive to social pressures of this kind. For specific empirical evidence about the effects that social considerations may have on educational choices see Cipollone and Rosolia (2007).

${ }^{2}$ Non-selfish agents are agents whose actions are influenced by the behaviour of some other individuals or reference group. See for instance Fehr and Fischbacher (2002).
} 
composition of society, the model endogenously displays a growing level of average education. For instance, education is likely to increase, even in a purely conformist society, provided that the average productivity level is not too low. However, the existence of agents with interdependent preferences, although necessary in the context considered, is not sufficient to trigger some positive educational dynamics. The model also rationalizes other stylized facts. In fact, as the level of average education increases, the signals that workers send to the labour market can become less informative and potentially harmful for the profitability of firms. Therefore, employers progressively become more demanding when setting their educational requirements, while myopic individuals who fail to anticipate this trend are disappointed by the wages they are offered.

The paper is organized as follows: Section 2 reviews the relevant literature; Section 3 introduces and solves a repeated signalling game enriched with concerns about social distance; Section 4 studies the dynamics of the average educational level in society; Section 5 analyses the behaviour of firms; Section 6 concludes.

\section{Related literature}

In this paper, we provide an explanation for the increase in education over the last few decades. This explanation is based on a signalling model enriched with theories of social distance and interdependent preferences. This section briefly reviews (some of) the relevant literature in these three areas.

(i) The increase in education There are various reasons that may help to explain the positive trend in the average levels of education attained in developed countries. A first and fairly obvious explanation is that people study more because by doing so they expect to receive higher wages. Indeed, the positive relationship between schooling and earnings is generally confirmed (see for instance Ashenfelter et al., 1999; and Psacharopoulos and Patrinos, 2004) by the estimation of Mincerian equations. ${ }^{3}$ Related to wage expectations is the literature that focuses on the effects that skill-biased technological progress has on the distribution of wages (see Heckman et al., 1998). The idea here is that the wage gap between unskilled and skilled workers has widened considerably in recent years, providing incentives for acquiring a higher level of education.

From a social point of view, education can be seen as an investment in human capital which has beneficial effects on economic growth (Barro, 2001). Indeed, many government policies have been designed with the purpose of raising the educational level in society. These policies include an increase and diversification in the supply of education, subsidies and other incentives and an increase in the years of compulsory schooling. On the demand side, individuals face a trade-off

\footnotetext{
${ }^{3}$ Jacob Mincer was the first one to empirically study the relation between schooling and labour income.

${ }^{4}$ Becker (1993) offers a deep analysis of the effects that education, training, and on-the-job experience have on an individual's human capital.
} 
between studying and working which is affected by labour market conditions. For instance, during periods of high unemployment, the opportunity cost of continuing in full-time education is lower. Empirical evidence about this relationship is given in Giannelli and Monfardini (2003) and Dellas and Sakellaris (2003).

(ii) Signalling The concept of signalling was introduced in the seminal paper by Spence (1973) and has since become an important branch of the so-called economics of information. Riley (2001) presents a rich overview of the applications of signalling models. Signalling is an attempt to solve problems of asymmetric information. In such a situation, the informed agent may have incentives to adopt behaviours (signals) that reveal some of his unobservable characteristics to the uninformed party. In Spence's model, the signal is the level of schooling an individual acquires. In fact, it is assumed that education conveys information about unobservable and innate productivity.

Signalling games often have multiple equilibria. In some of these equilibria, individuals with different characteristics send different signals (separating equilibria); in others, they adopt the same behaviour such that signals are uninformative (pooling equilibria). Various concepts of equilibrium refinements have been proposed. In Section 3.1, we will apply the most common one, namely, the intuitive criterion due to Cho and Kreps (1987).

(iii) Social distance and interdependent preferences Our daily lives provide plenty of evidence in support of the hypothesis of interdependence of individuals' preferences. Indeed, allowing for interdependent preferences involves acknowledging that sentiments, like pride, esteem, shame or acceptance, are important in agents' choices. The investigation of these issues began with early studies of consumption patterns of individuals. For example, Duesenberry (1949) includes the average level of consumption into the utility function that individuals have to maximize so that an agent increases his utility if he is able to 'beat the average'. A slightly different methodology was followed by Pollak (1976), who models preferences as depending upon other people's past consumption. This setting leads to non-strategic behaviour by agents and the model becomes analytically more tractable. From a more theoretical point of view, the incentives that move status-seeking individuals were described first by Frank (1985). Robson (1992) considers the situation in which people are interested in the ordinal rank they occupy in the distribution of wealth, while Hopkins and Kornienko (2004) study the case in which utility is affected by the amount of general consumption, as well as by the consumption of a particular good which defines the status.

When preferences are defined by the relative amount of consumption or wealth, it is natural to assume a tendency towards status-seeking behaviour. Yet, in other contexts, a more conformist attitude may be the rule rather than the exception. The classic work on conformism by Jones (1984) studied examples of social influences in a college environment, in the army and in the workplace. Focusing on the last example, Jones analysed the effort workers decide to exert. Given that extreme behaviours are stigmatized, the slowest (fastest) members of the working group 
feel the pressure to speed up (slow down). Moreover, new workers imitate the behaviour of older colleagues. Evidence for these forms of peer pressures also comes from experiments (Falk and Ichino, 2006), while a more theoretical analysis of the tendency towards conformism is given by Bernheim (1994).

To sum up, both status-seeking and conformist individuals care about the social distance between them and some reference group. Externalities arise in both cases and the social efficiency of the final outcome is not ensured. Using simple formulations of utility functions, Akerlof (1997) showed that status-seeking behaviour usually leads to overindulgence in the status-producing activity (i.e., overconsumption). ${ }^{5}$ The outcome deriving from conformist behaviour can instead range from underprovision to overprovision.

\section{The model}

\subsection{A basic signalling model}

Consider a population of potential workers. ${ }^{6}$ A proportion $\alpha_{h} \in(0,1)$ is characterized by high productivity $\left(\theta_{h}=2\right)$, while the remaining proportion $\left(\alpha_{1}=1-\alpha_{h}\right)$ has low productivity $\left(\theta_{l}=1\right)$. At least two firms compete in hiring the workers. These firms are not able to distinguish between the two categories, based on the agents' observable characteristics. Thus, if no signals are available, firms offer a wage equal to average productivity $\bar{w}=\bar{\theta}=2 \alpha_{h}+\alpha_{l}$, with $\bar{w} \in(1,2)$. A more efficient outcome can be achieved through signalling.

Assume that firms have some beliefs about the productivity of workers, i.e., they think that there exists a certain level of education $(\tilde{e})$ such that if a worker $i$ acquires a level of education $e_{i} \geqslant \tilde{e}$, then he must be highly productive. Conversely if $e_{i}<\tilde{e}$ it must be the case that the individual has low productivity. Given these beliefs and the specific costs of education (high types have a cost which is half the cost of low types), the utility functions of the workers take the following form:

$$
U_{h}(e)=2-\frac{1}{2} e^{2} \quad U_{l}(e)=1-e^{2}
$$

Optimal educational levels must be subject to two incentive compatibility constraints which require high productivity individuals not to have any incentive to pretend to be low productivity types and vice-versa:

$$
2-\frac{1}{2} \tilde{e}^{2} \geqslant 1 \quad 1 \geqslant 2-\tilde{e}^{2}
$$

The constraints are satisfied by any $\tilde{e} \in[1, \sqrt{2}]$. For any level of $\tilde{e}$ in the interval, the types of agents send different signals (we restrict our attention to

\footnotetext{
${ }^{5}$ In an older paper, Akerlof (1976) describes the so-called rat-race mechanism: the probability of winning a prize increases in the effort agents exert such that this results in an overprovision of effort.

${ }^{6}$ The model is almost identical to the one introduced by Spence (1973) and provides the starting point for a richer framework that we introduce in the next section.
} 
separating equilibria). In particular low productivity workers choose $e_{l}=0$ and high productivity workers choose $e_{h}=\tilde{e}$. Among all these perfect Bayesian equilibria, the intuitive criterion (Cho and Kreps, 1987) selects the least cost-separating equilibrium: low productivity workers get the minimum level of education $\left(e_{l}^{*}=0\right)$, while high productivity workers choose $e_{h}^{*}=1$. This is the lowest possible level of education which cannot be profitably mimicked by the low types. It follows that the average level of education is given by $\bar{e}=\alpha_{h}$.

\subsection{A richer framework: signalling and social distance}

Spence's signalling game models the choices of a single generation of workers. Therefore, its results are essentially static. Results remain static even when considering a repeated version of the game (subsequent cohorts of workers facing the oneshot signalling game). In fact, assuming that the average productivity is equal among different generations, the average level of education would remain constant over time at the level $\bar{e}_{t}=\alpha_{h}$. In the standard model, firms' beliefs match workers' actual productivity such that neither workers nor firms have any incentive to modify their strategies. ${ }^{7}$

We study a richer framework that may display some dynamics. In our game, two subsequent cohorts of individuals participate in a signalling game. Every cohort is formed by many agents and every agent is characterized by a certain level of productivity and some social preferences. Both the productivity level and social preferences are assumed to be innate and fixed. In fact, prior to any decision of any player, two simultaneous moves of Nature determine the composition of each cohort. As in the standard model, agents are split into two classes according to their (low or high) productivity. The other move of Nature defines the social preferences with respect to the average education in society. Some individuals, possibly the majority, simply do not have social preferences (lower index $i$ for independent) and are therefore analogous to the agents in Spence's model; some are characterized by a conformist behaviour (lower index $c$ ); some are statusseeking (lower index $s$ ).

Each cohort of potential workers, whose size is normalized to 1 , is thus partitioned as shown in Table 1. Obviously $\alpha_{k j} \in[0,1]$ for any $k \in\{i, c, s\}$ and any $j \in\{l, h\}$ and $\sum_{k} \sum_{j} \alpha_{k j}=1$. The two cohorts are identical (same size and same $\alpha_{k j}$ ). Therefore, any movement in the average level of education will be endogenously generated by the model and will not be due to population growth, changes in the productivity level or in the social preferences of the agents.

Once Nature has moved, the following occurs:

$t=1$ : The first cohort of workers plays the signalling game.

$t=2$ : The second cohort of workers plays the signalling game.

\footnotetext{
${ }^{7}$ Indeed the original paper postulates self-confirming beliefs of the firms exactly to avoid studying a non stationary system (Spence, 1973, p.360).
} 
Table 1 The composition of each cohort of individuals

Low productivity $(l)$

High productivity $(h)$

$\begin{array}{llc}\text { Independent }(i) & \alpha_{i l} & \alpha_{i h} \\ \text { Conformist }(c) & \alpha_{c l} & \alpha_{c h} \\ \text { Status-seeking }(s) & \alpha_{s l} & \alpha_{s h}\end{array}$

We are interested in studying the dynamics of the average level of education. We indicate with $\bar{e}_{t}$ the weighted average of the levels of education chosen by each class at time $t$.

$$
\bar{e}_{t}=\sum_{k} \sum_{j} \alpha_{k j} e_{k j}^{t} \quad k \in\{i, c, s\}, j \in\{l, h\}
$$

Before proceeding to the main analysis, we introduce and discuss a fundamental assumption of the model: workers are myopic, while firms have perfect foresight. ${ }^{8}$

The idea is that every single worker 'plays the game only once'. In other words, workers are inexperienced players who may lack all the relevant information or may have problems in processing it properly. Therefore, their decision about the level of education to acquire is likely to be based on simplifying assumptions. In contrast, firms are more sophisticated players, are constantly in the game and do not suffer from these problems. Following on from some important recent contributions in the behavioural literature, such an approach (myopic individuals interacting with sophisticated firms) is one that is increasingly used in economics. ${ }^{9}$

In the context of our model, the myopia of workers means that individuals, in choosing at time $t$ their educational level at $t+1$, assume that the situation of the education/labour market at time $t+1$ will be analogous to the situation observed at time $t$.

This has two implications. First, agents observe $\tilde{e}_{t}$ (the current level of education that firms use to discriminate between high and low productivity individuals) and assume that it will remain constant; therefore, they set $E_{t}\left(\tilde{e}_{t+1}\right)=\tilde{e}_{t}$. Second, nonselfish agents (conformists and status-seekers) also set $E_{t}\left(\bar{e}_{t+1}\right)=\bar{e}_{t}$, i.e., they evaluate their relative position using as a benchmark the current average educational level and not the (possibly different) one that will arise at the time they enter into the labour market. ${ }^{10}$

\footnotetext{
${ }^{8}$ The Appendix shows how the results of the model change under the alternative and more traditional assumption of both the workers and the firms being fully rational.

${ }^{9}$ For instance, it underpins the analysis of so-called behavioural industrial organization where rational firms face boundedly rational consumers (Oster and Scott Morton, 2005; Gabaix and Laibson, 2006).

${ }^{10}$ This approach is common in the literature that considers intertemporal effects of interdependent preferences (Pollak, 1976; Jones, 1984). Focusing on educational choices it is used, among others, by Freeman (1971), Manski (1993) and Wilson et al. (2005). Manski (1993, p.49) writes: 'youths observe the incomes realized by members of the preceding generation who chose schooling, and they make inferences from these observations'.
} 
Such myopic behaviour seems to be common in practice. Indeed, it can still provide a decently precise approximation if average levels of education move slowly over time or if the time gap between today's decision of enrolling in a certain degree and tomorrow's entry in the labour market is short. For instance, a student considering the idea of taking a MBA will use the current average level of education to evaluate his future relative position. Another, more practical, consideration that justifies the myopic assumption is based on how people become informed about the available educational choices and current labour market conditions. The usual sources of information are provided by comments of (older) friends and by reading student guides and statistics about the likelihood of getting a job with a certain degree. All of these pieces of information describe the current situation, not the future.

Finally, this kind of myopic behaviour is also confirmed by a recent paper (Brunello et al., 2004) which studies wage expectations of European college students. The study shows that these expectations are substantially higher than the actual wages. In other words, players hold incorrect expectations, i.e., they are myopic. ${ }^{11}$ People often admit to being disappointed by the wage they get conditional on their level of schooling. This would not happen if individuals were fully rational. ${ }^{12}$

We assume however that firms are sophisticated and forward-looking such that they always adopt an optimal screening policy. Therefore, in the first period (standard signalling game), they offer the wage $w=1$ to any individual whose educational level is such that $0 \leqslant e^{*}<1$; they offer $w=2$ to all agents with $e^{*} \geqslant 1$. But in the second period they may update their screening policy if they realize that, because of the noise caused by social distance considerations, the critical value of $\tilde{e}=1$ is no more effective in discriminating among workers with different levels of productivity.

\subsection{Time $t=1$}

At the beginning of the first period, individuals' choices are not influenced by social preferences because of the absence of a current average level of education with which to make a comparison. This stage of the game is analogous to the standard signalling model presented in Section 3.1. It provides a starting point upon which the model develops in $t=2$. Low productivity workers attain a level of education equal to 0 , while high productivity individuals choose a level of 1 . Optimal levels of education are thus $e_{i l}^{*}=e_{c l}^{*}=e_{s l}^{*}=0$ and $e_{i h}^{*}=e_{c h}^{*}=e_{s h}^{*}=1$. The average at the end of $t=1$ is $\bar{e}_{1}=\alpha_{i h}+\alpha_{c h}+\alpha_{\text {sh }}$.

\footnotetext{
${ }^{11}$ Manski (2004) explicitly considers the effect of wage expectations on schooling choices. As he writes (Manski, 2004, p.1336): 'If experts disagree on the returns to schooling, is it plausible that youth have rational expectations? I think not'.

${ }^{12}$ An anonymous referee correctly pointed out that there is also a 'statistical' explanation for this regularity. Assume wages are stochastic and workers expect to receive the average wage. Then the majority of them will be disappointed given that wages distributions are usually distorted such that the median wage is lower than the average wage.
} 
Table 2 Utility functions of the six classes of individuals

\begin{tabular}{|c|c|c|}
\hline & Low productivity & High productivity \\
\hline $\begin{array}{l}\text { Independent } \\
\text { Conformist }\end{array}$ & $\begin{array}{l}U_{i l}=w-e^{2} \\
U_{c l}=w-e^{2}-\left(e-\bar{e}_{1}\right)^{2}\end{array}$ & $\begin{array}{l}U_{i h}=w-\frac{1}{2} e^{2} \\
U_{c h}=w-\frac{1}{2} e^{2}-\left(e-\bar{e}_{1}\right)^{2}\end{array}$ \\
\hline Status-seeking & $\begin{cases}U_{s l}=w-e^{2}-2\left(e-\bar{e}_{1}\right)^{2} & \text { if } e<\bar{e}_{1} \\
U_{s l}=w-e^{2}+2 \bar{e}_{1}\left(e-\bar{e}_{1}\right) & \text { if } e \geqslant \bar{e}_{1}\end{cases}$ & $\begin{cases}U_{s h}=w-\frac{1}{2} e^{2}-2\left(e-\bar{e}_{1}\right)^{2} & \text { if } e<\bar{e}_{1} \\
U_{s h}=w-\frac{1}{2} e^{2}+2 \bar{e}_{1}\left(e-\bar{e}_{1}\right) & \text { if } e \geqslant \bar{e}_{1}\end{cases}$ \\
\hline
\end{tabular}

\subsection{Time $t=2$}

At the beginning of the second period, a new cohort of potential workers faces their educational choice. The choices of non-selfish players are now influenced by their specific social preferences. Table 2 reports the utility functions of the six categories of agents (subscripts are omitted whenever unnecessary). Wages appear in the implicit form $w$ as they depend on the individuals' actual educational choices. In particular, because of the assumption of myopia discussed in Section 3.2, workers expect to receive the low wage $w=1$ whenever they choose $e^{*}<1$, while they expect to receive the high wage $w=2$ whenever $e^{*} \geqslant 1$.

Independent players are characterized by standard utility functions à la Spence. Utility functions for the players with interdependent preferences are slightly more complex. Besides the part which captures the trade-off between wages and the costs of education, there is an extra term that models social preferences. In accordance with many classical contributions in the field (see Pollak, 1976; Jones, 1984; Bernheim, 1994; Akerlof, 1997), this last term enters additively into utility functions. For the reasons which have been explained in Section 3.2, this social component is a function of $\bar{e}_{1}$, the current average level that workers of the second cohort observe when deciding the level of education that they will have in $t=2$. In line with the utility functions presented in Spence's original article (1973), this additional part appears as an explicit function which is kept as simple as possible.

Conformist individuals have a preference for being close to the average. The social term in the utility function assigns an increasing cost whenever their educational choice differs from the average level $\bar{e}_{1}$. The utility functions of status-seeking individuals are more problematic. A distinction has to be drawn between two cases. If the level of education is below $\bar{e}_{1}$, then status ambitions are frustrated. In this case, the utility function resembles those of conformist individuals but with a higher cost for falling behind. However if the educational level is above $\bar{e}_{1}$, then the individual's utility increases in this distance. In this second case, the function has to be corrected in some way. Consider, for example, the sh-class. With respect to the case of $e<\bar{e}_{1}$, the symmetric formulation for $e \geqslant \bar{e}_{1}$ would be $U_{s h}=w-\frac{1}{2} e^{2}+2\left(e-\bar{e}_{1}\right)^{2}$. However, this is a convex function $\left(\frac{\partial^{2} U_{s h}}{\partial e^{2}}=3\right)$ such that the first order condition would identify a minimum and not a maximum. To have a meaningful solution, the utility function takes the form presented in Table 2. 


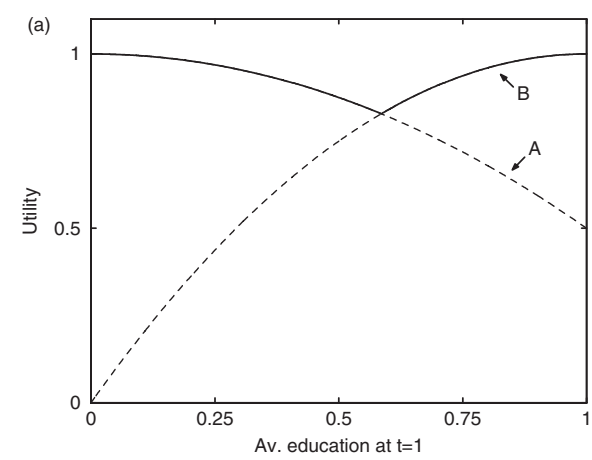

Fig. 1. (a) Utility of the $c l$-class (b) Education of the $c$-class

Notice that the positive effect of an increasing distance $\left(e-\bar{e}_{1}\right)$ is amplified if the average level in $t=1$ is high.

We already know from Section 3.1 that the optimal choices of individuals with independent preferences are $e_{i l}^{*}=0$ and $e_{i h}^{*}=1$. The following subsections study the behaviour of the other four classes of agents.

3.4.1 The 'conformist + low productivity' class (cl) Cl-individuals face the problem $\max U_{c l}=w-e^{2}-\left(e-\bar{e}_{1}\right)^{2}$ whose solution is given by $\hat{e}_{c l}=\frac{1}{2} \bar{e}_{1}$. Since $\bar{e}_{1} \in[0,1]$ it follows that $\hat{e}_{c l} \in\left[0, \frac{1}{2}\right]$. Given that $\hat{e}_{c l}<1$, agents expect to receive $w=1$ because firms correctly categorize them as low types. Is it always optimal to choose $\hat{e}_{c l}$ ? A possible alternative for a $c l$-agent is to choose $e_{c l}=1$ in which case, despite the higher educational costs, the agent expects to receive the high wage $w=2$. The following figures provide a graphical analysis of the situation.

$$
\begin{array}{ll}
\text { A) } U_{c l}\left(\frac{1}{2} \bar{e}_{1}\right)=1-\frac{1}{2}\left(\bar{e}_{1}\right)^{2} & \text { B) } U_{c l}(1)=-\left(\bar{e}_{1}\right)^{2}+2 \bar{e}_{1}
\end{array}
$$

Figure 1a plots the two utility functions for $\bar{e}_{1} \in[0,1]$. The optimal level of education $e_{c l}^{*}$ (Fig. 1b) is then derived from the upper contour set of the functions $A$ and $B$. Up to a critical value ( $\overline{\mathrm{e}}_{1} \cong 0.586$ ), it is optimal to choose $e_{c l}^{*}=\frac{1}{2}$. Above the threshold it is instead more convenient to 'jump' to $e_{c l}^{*}=1$. The intuition for such behaviour is clear. As long as the average level is low, a $c l$-agent can conform to it without investing too much in costly education. But if $\bar{e}_{1}$ is high enough, the incentives to choose $e_{c l}^{*}=1$ become dominant as the agent expects to receive the high wage $w=2$.

3.4.2 The 'conformist + high productivity' class (ch) Workers belonging to the ch-class have to solve $\max U_{c h}=w-\frac{1}{2} e^{2}-\left(e-\bar{e}_{1}\right)^{2}$. The solution is given by $\hat{e}_{c h}=\frac{2}{3} \bar{e}_{1}$. Given that $\hat{e}_{c h} \stackrel{e}{\in}\left[0, \frac{2}{3}\right]$, the expected wage is $w=1$. On the other hand, by choosing $e_{c h}=1$, a $c h$-agent expects to receive $w=2$. Figure 2a compares the utilities arising from these two alternative choices.
A) $U_{c h}\left(\frac{2}{3} \bar{e}_{1}\right)=1-\frac{1}{3}\left(\bar{e}_{1}\right)^{2}$
B) $U_{c h}(1)=\frac{1}{2}-\left(\bar{e}_{1}\right)^{2}+2 \bar{e}_{1}$ 


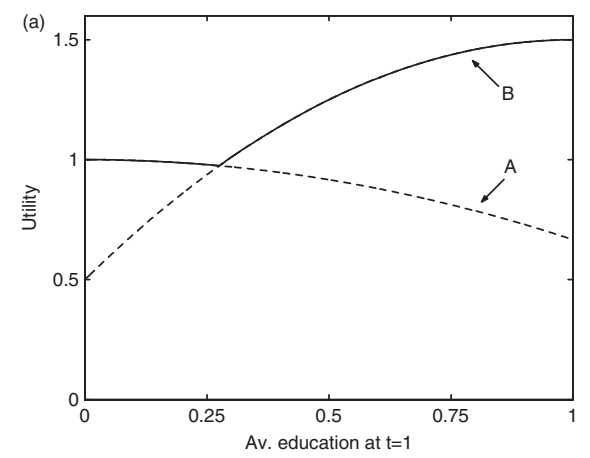

Fig. 2. (a) Utility of the ch-class (b) Education of the ch-class

Figure $2 \mathrm{~b}$ shows that the optimal strategy is to choose $e_{c h}^{*}=1$ as long as the average level in $t=1$ is not too low (the critical value is $\bar{e}_{1} \cong 0.275$ ). By doing so, agents are effectively signaling their high productivity. Below the critical value, it is instead more convenient to choose $e_{c h}^{*}=\frac{2}{3} \bar{e}_{1}$. When the average is very low, pressures to conform are stronger than incentives to signal actual productivity. Notice that the $c h$-class and the $c l$-class may be pooled at $e^{*}=1$.

3.4.3 The 'status-seeking + low productivity' class (sl) The analysis of the behaviour of status-seeking agents is more complex given that two different utility functions have to be considered (see Table 2). Consider the case of a $s$-agent. If the optimal choice is below $\bar{e}_{1}$ then the problem is $\max U_{s l}=w-e^{2}-2\left(e-\bar{e}_{1}\right)^{2}$ which has soluztion $\hat{e}_{s l}=\frac{2}{3} \bar{e}_{1}$. Otherwise, the problem is $\max _{e} U_{s l}=w-e^{2}+2 \bar{e}_{1}\left(e-\bar{e}_{1}\right)$ which leads to $\hat{e}_{s l}=\bar{e}_{1}$. In both cases the worker expects to receive the low wage $w=1$.

The comparison between these two educational choices and the other relevant alternative $\left(e_{s l}=1\right)$ is depicted in Fig. 3a. Figure 3b displays the optimal level of education of $s l$-agents.
A) $U_{s l}\left(\frac{2}{3} \bar{e}_{1}\right)=1-\frac{2}{3}\left(\bar{e}_{1}\right)^{2}$
B) $U_{s l}\left(\bar{e}_{1}\right)=1-\left(\bar{e}_{1}\right)^{2}$
C) $U_{s l}(1)=1-2\left(\bar{e}_{1}\right)^{2}+2 \bar{e}_{1}$

Status-seeking individuals with low productivity always choose $e_{s l}^{*}=1$. The reason is that in the original Spence model (see Section 3.1), low type individuals are indifferent between $e_{s l}^{*}=0$ and $e_{s l}^{*}=1$. The least cost-separating equilibrium selects $e_{s l}^{*}=0$. In our model, $s l$-individuals that choose $e_{s l}^{*}=1$ in $t=2$ surely enjoy some extra status-related utility. This additional factor makes $e_{s l}^{*}=1$ a dominant strategy.

3.4.4 The 'status-seeking + high productivity' class (sh) Two different situations have to be considered also for the $s h$-class (See Table 2). For educational choices that are smaller than $\bar{e}_{1}$, the problem is given by $\max U_{s h}=w-\frac{1}{2} e^{2}-2\left(e-\bar{e}_{1}\right)^{2}$. The optimal choice is $\hat{e}_{s h}=\frac{4}{5} \bar{e}_{1}$ which implies $w=1$. For values that are above $\bar{e}_{1}$, the appropriate utility function is $U_{s h}=w-\frac{1}{2} e^{2}+2 \bar{e}_{1}\left(e-\bar{e}_{1}\right)$. This function is maximized by $\hat{e}_{s h}=2 \bar{e}_{1}$ such that an sh-agent expects $w=1$ if $\bar{e}_{1}<\frac{1}{2}$ and $w=2$ if $\bar{e}_{1} \geqslant \frac{1}{2}$. 


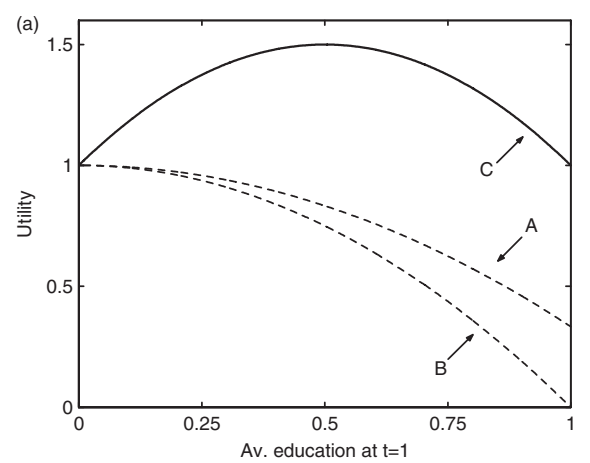

Fig. 3. (a) Utility of the sl-class

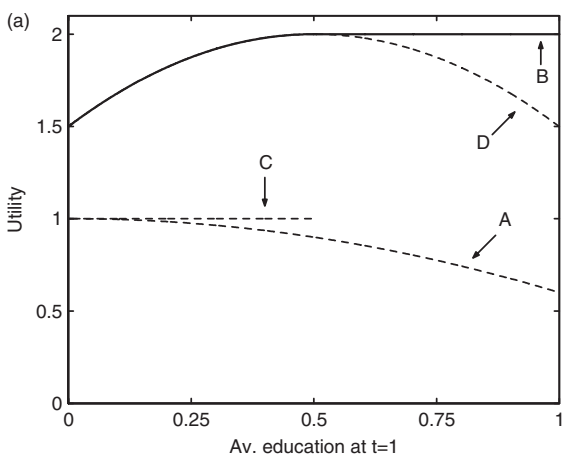

Fig. 4. (a) Utility of the sh-class (b) Education of the sh-class

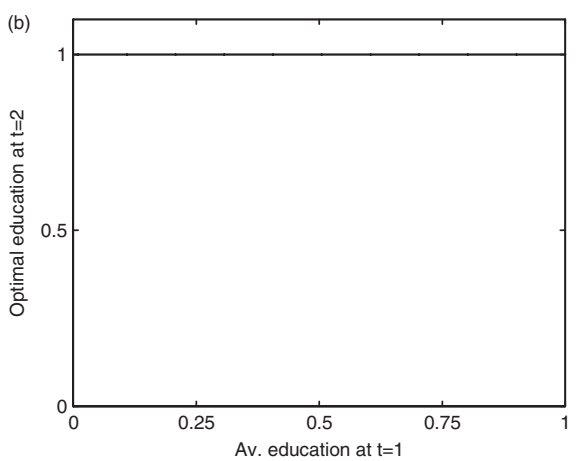

(b) Education of the sl-class

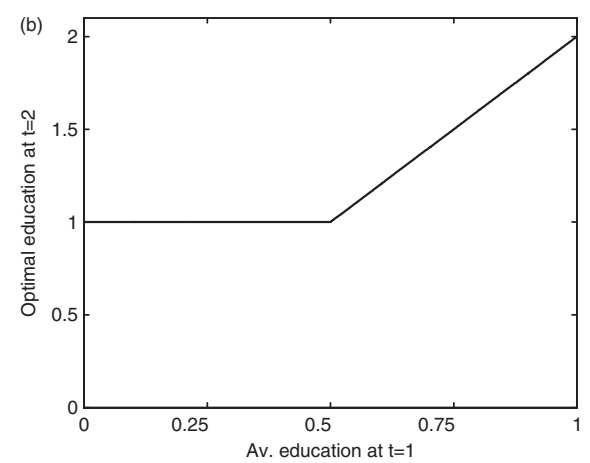

Figures $4 \mathrm{a}$ and $4 \mathrm{~b}$ illustrate the relevant utility functions and the optimal choice $e_{s h}^{*}$.
A) $U_{s h}\left(\frac{4}{5} \bar{e}_{1}\right)=1-\frac{2}{5}\left(\bar{e}_{1}\right)^{2}$
C) $U_{s h}\left(2 \bar{e}_{1}\right)=1$ with $\bar{e}_{1}<\frac{1}{2} \%$
B) $U_{s h}\left(2 \bar{e}_{1}\right)=2$ with $\bar{e} \%_{1} \geqslant \frac{1}{2}$
D) $U_{s h}(1)=\frac{3}{2}-2\left(\bar{e}_{1}\right)^{2}+2 \bar{e}_{1} \%$

As long as $\overline{\mathrm{e}}_{1} \leqslant 0.5$, a $s h$-player chooses $e_{s h} \%^{*}=1$ given that the distance between his choice and the average level is high enough to satisfy his status aspirations. For any $\bar{e}_{1}>0.5$, the player chooses $e_{s h}^{*}=2 \bar{e}_{1}$. In this second case, the agent realizes he needs a higher level of education in order to reach a satisfying status.

\section{Education dynamics}

Having solved for agents' optimal behaviour, it is possible to focus on $\bar{e}_{2}$, the average level of education at time $t=2$. The following table summarizes the behaviour of the six classes of individuals. We use the letter $\lambda$ to indicate the critical value at which players' optimal choices display discontinuities. 
Table 3 Optimal choices at $t=1$ and at $t=2$

\begin{tabular}{ccccccc}
\hline Class & Prop. & Product. $\boldsymbol{\theta}$ & $\boldsymbol{e}_{1}^{*}$ & $\boldsymbol{e}_{2}^{*}$ for $\overline{e_{1}} \leqslant \boldsymbol{\lambda}$ & $\boldsymbol{e}_{2}^{*}$ for $\overline{e_{1}}>\boldsymbol{\lambda}$ & $\boldsymbol{\lambda}$ \\
\hline$i l$ & $\alpha_{i l}$ & 1 & 0 & 0 & 0 & - \\
$i h$ & $\alpha_{i h}$ & 2 & 1 & 1 & 1 & - \\
$c l$ & $\alpha_{c l}$ & 1 & 0 & $\frac{1}{2} \bar{e}_{1}$ & 1 & 0.586 \\
$c h$ & $\alpha_{c h}$ & 2 & 1 & $\frac{2}{3} \bar{e}_{1}$ & 1 & 0.275 \\
$s l$ & $\alpha_{s l}$ & 1 & 0 & 1 & 1 & - \\
$s h$ & $\alpha_{s h}$ & 2 & 1 & 1 & $2 \overline{e_{1}}$ & 0.500 \\
\hline
\end{tabular}

The average level of education is given by $\bar{e}_{t}=\sum_{k} \sum_{j} \alpha_{k j} e_{k j}^{t}$ for $k \in\{i, c, s\}$ and $j \in\{l, h\}$ such that $\bar{e}_{1}=\alpha_{i h}+\alpha_{c h}+\alpha_{\text {sh }}$.

Notice that the two cohorts of players with independent preferences ( $i l$ and $i h$ ) are characterized by a constant level of education over the entire game. The model therefore predicts that the average level of education will remain constant in a society where no player is influenced by issues of social distance in educational achievements. This would actually be the case of a standard signalling game repeated over time.

Proposition 1 The presence of individuals with interdependent preferences is a necessary condition for observing an average level of education that changes over time.

Proof If there are no agents with interdependent preferences then $\alpha_{i l}+\alpha_{i h}=1$. Optimal educational choices are $e_{i l}^{*}=0$ and $e_{i h}^{*}=1$ both in $t=1$ and in $t=2$. It follows that $\bar{e}_{2}=\bar{e}_{1}=\alpha_{i h}$.

Therefore, the average level of education can change only if optimal choices of the four classes of individuals with interdependent preferences differ between different periods. The average level of education in $t=2$ is a weighted average of the optimal choices by the six classes of individuals. These choices are functions of $\bar{e}_{1}$. Moreover, $\bar{e}_{1}$ is a function of the $\alpha_{k j}$, i.e., the proportions of the different classes. It follows that $\bar{e}_{2}$ is a discontinuous function of the $\alpha_{k j}$. More precisely, $\bar{e}_{2}$ takes the following form:

$$
\bar{e}_{2}= \begin{cases}\alpha_{i h}+\alpha_{c l}\left(\frac{1}{2} \bar{e}_{1}\right)+\alpha_{c h}\left(\frac{2}{3} \bar{e}_{1}\right)+\alpha_{s l}+\alpha_{s h} & 0.000 \leqslant \bar{e}_{1} \leqslant 0.275 \\ \alpha_{i h}+\alpha_{c l}\left(\frac{1}{2} \bar{e}_{1}\right)+\alpha_{c h}+\alpha_{s l}+\alpha_{s h} & 0.275<\bar{e}_{1} \leqslant 0.500 \\ \alpha_{i h}+\alpha_{c l}\left(\frac{1}{2} \bar{e}_{1}\right)+\alpha_{c h}+\alpha_{s l}+\alpha_{s h}\left(2 \bar{e}_{1}\right) & 0.500<\bar{e}_{1} \leqslant 0.586 \\ \alpha_{i h}+\alpha_{c l}+\alpha_{c h}+\alpha_{s l}+\alpha_{s h}\left(2 \bar{e}_{1}\right) & 0.586<\bar{e}_{1} \leqslant 1.000\end{cases}
$$

The study of this function in its general form is not feasible because it involves too many unknowns. Therefore, we focus on some special cases that are still useful in understanding the effects that social preferences have on educational dynamics. In particular, we study the cases in which, in addition to the two classes characterized by independent preferences (possibly covering the vast majority of the 
population), there are also some specific combinations of classes of individuals with interdependent preferences.

\subsection{Subpopulation with homogeneous social preferences}

4.1.1 Independent and conformist individuals A society with independent and conformist individuals implies the restrictions $\alpha_{i l}+\alpha_{i h}+\alpha_{c l}+\alpha_{c h}=1$ and $\bar{e}_{1}=\alpha_{i h}+\alpha_{c h}$. The function $\bar{e}_{2}$ and, most importantly, the difference in the average educational levels between the two periods $\left(\Delta \bar{e}=\bar{e}_{2}-\bar{e}_{1}\right)$ are:

$$
\bar{e}_{2}=\left\{\begin{array}{l}
\alpha_{i h}+\alpha_{c l}\left(\frac{1}{2} \bar{e}_{1}\right)+\alpha_{c h}\left(\frac{2}{3} \bar{e}_{1}\right) \\
\alpha_{i h}+\alpha_{c l}\left(\frac{1}{2} \bar{e}_{1}\right)+\alpha_{c h} \\
\alpha_{i h}+\alpha_{c l}+\alpha_{c h}
\end{array} \quad \Delta \bar{e}= \begin{cases}\alpha_{c l}\left(\frac{1}{2} \bar{e}_{1}\right)+\alpha_{c h}\left(\frac{2}{3} \bar{e}_{1}-1\right) & 0.000 \leqslant \bar{e}_{1} \leqslant 0.275 \\
\alpha_{c l}\left(\frac{1}{2} \bar{e}_{1}\right) & 0.275<\bar{e}_{1} \leqslant 0.586 \\
\alpha_{c l} & 0.586<\bar{e}_{1} \leqslant 1.000\end{cases}\right.
$$

In line with Proposition 1 , the change in the average level of education $(\Delta \bar{e})$ is solely a function of the share of conformist individuals. At the same time, a closer inspection of $\Delta \bar{e}$ provides an example which allows us to state the following proposition:

Proposition 2 The presence of individuals with interdependent preferences is not sufficient for observing an average level of education that changes over time.

Proof In a population such that $\alpha_{i l}+\alpha_{i h}+\alpha_{c l}+\alpha_{c h}=1$, consider the interval $\bar{e}_{1} \in[0,0.275]$ and set $\Delta \bar{e}=0$ (constant level of average education), i.e., $\alpha_{c l}\left(\frac{1}{2} \bar{e}_{1}\right)+\alpha_{c h}\left(\frac{2}{3} \bar{e}_{1}-1\right)=0$. This last condition holds if $\tilde{\alpha}_{c l}=\gamma \tilde{\alpha}_{c h}$ where $\gamma=\frac{1-\frac{2}{3} \bar{e}_{1}}{\frac{1}{2} \bar{e}_{1}}$. Shares like $\tilde{\alpha}_{c l}$ and $\tilde{\alpha}_{c h}$ identify a stationary outcome of the game.

Proposition 2 completes the statement of Proposition 1: Interdependent preferences are a necessary but not sufficient condition in order to observe a level of average education that changes over time. We now study the conditions under which the average level of education increases. These conditions turn out not to be particularly demanding. Education is likely to increase in a society constituted only of independent and conformist individuals. In particular, it increases if at least $27.5 \%$ of the population is highly productive.

Proposition 3 Average education can increase even in a society with no statusseeking individuals. In particular:

(a) education increases for any $\bar{e}_{1}>0.275$, provided that there is at least one conformist individual with low productivity;

(b) education can either increase or decrease for any $\bar{e}_{1} \leqslant 0.275$.

Proof If there are no status seeking individuals then $\alpha_{i l}+\alpha_{i h}+\alpha_{c l}+\alpha_{c h}=1$ and $\bar{e}_{1}=\alpha_{i h}+\alpha_{c h}$. Then:

(a) for any $\bar{e}_{1} \in(0.275,1]$ the difference between $\bar{e}_{1}$ and $\bar{e}_{2}$ (see the formulation of $\Delta \bar{e}$ above) is either $\Delta \bar{e}=\alpha_{c l}\left(\frac{1}{2} \bar{e}_{1}\right)$ or $\Delta \bar{e}=\alpha_{c l}$. In both cases $\Delta \bar{e}>0$ if $\alpha_{c l}>0$; 
(b) for any $\bar{e}_{1} \in[0,0.275]$ the difference between $\bar{e}_{1}$ and $\bar{e}_{2}$ is given by $\Delta \bar{e}=\alpha_{c l}\left(\frac{1}{2} \bar{e}_{1}\right)+\alpha_{c h}\left(\frac{2}{3} \bar{e}_{1}-1\right)$. The proof of Proposition 2 shows that shares such that $\tilde{\alpha}_{c l}=\gamma \tilde{\alpha}_{c h}$ identify stationary outcomes of the game. It follows that average education increases if $\alpha_{c l}>\tilde{\alpha}_{c l}$ and decreases if $\alpha_{c l}<\tilde{\alpha}_{c l}$.

Notice that we do not use the terminology 'equilibrium' to refer to the results of the game, since we have not analysed the behaviour of the other category of players, namely the firms. Section 5 will elaborate more on this point. To analyse the properties of the 'outcomes' described in the propositions, we introduce the following definition:

Definition 1 An outcome of the signalling game is called: perfectly separating if $e_{k l}^{*} \neq e_{y h}^{*}$ for any $k \in\{i, c, s\}$ and any $y \in\{i, c, s\}$; partly separating if $e_{k l}^{*}=e_{y h}^{*}$ for some $k \in\{i, c, s\}$ and some $y \in\{i, c, s\}$.

In a perfectly separating outcome (like the equilibrium of the game in $t=1$ ), players with different levels of productivity send different signals, i.e., they choose different levels of education. This is often not the case in $t=2$. Maintaining the focus on a population with only independent and conformist individuals, consider for instance the case in which $\bar{e}_{1}=0.6$. Optimal choices in $t=2$ (see Table 3 ) are $e_{i l}^{*}=0, e_{i h}^{*}=1, e_{c l}^{*}=1$ and $e_{c h}^{*}=1$. This is a partly separating outcome. Conformist individuals with low productivity choose $e_{c l}^{*}=1$, the same signal sent by high productivity classes (ih and $c h$ ). Partly separating outcomes easily arise in populations where conformist and/or status-seeking individuals are present.

The model, therefore, shows that the presence of agents with interdependent preferences can turn perfectly separating outcomes into partly separating ones. Or, in other words, social distance considerations add noise to an otherwise perfectly separating equilibrium. Section 5 analyses how the hiring policies of firms may be affected by this effect.

4.1.2 Independent and status-seeking individuals The presence of status-seeking individuals can surely generate some positive educational dynamics. Consider a population made of independent and status-seeking agents such that $\alpha_{i l}+\alpha_{i h}+$ $\alpha_{s l}+\alpha_{s h}=1$ and $\bar{e}_{1}=\alpha_{i h}+\alpha_{s h}$. The average level of education in $t=2$ and the difference with respect to $\bar{e}_{1}$ are given by:

$$
\bar{e}_{2}=\left\{\begin{array}{ll}
\alpha_{i h}+\alpha_{s l}+\alpha_{s h} \\
\alpha_{i h}+\alpha_{s l}+\alpha_{s h}\left(2 \bar{e}_{1}\right)
\end{array} \quad \Delta \bar{e}= \begin{cases}\alpha_{s l} & 0.000 \leqslant \bar{e}_{1} \leqslant 0.500 \\
\alpha_{s l}+\alpha_{s h}\left(2 \bar{e}_{1}-1\right) & 0.500<\bar{e}_{1} \leqslant 1.000\end{cases}\right.
$$

Notice that, also in this case, signals can be misleading and a partly separating outcome arises in $t=2$ : status-seeking agents with low productivity are pooled together with highly productive workers. The analysis of $\Delta \bar{e}$ directly leads to the following proposition.

Proposition 4 Education cannot decrease in a society where there are no conformist individuals. 


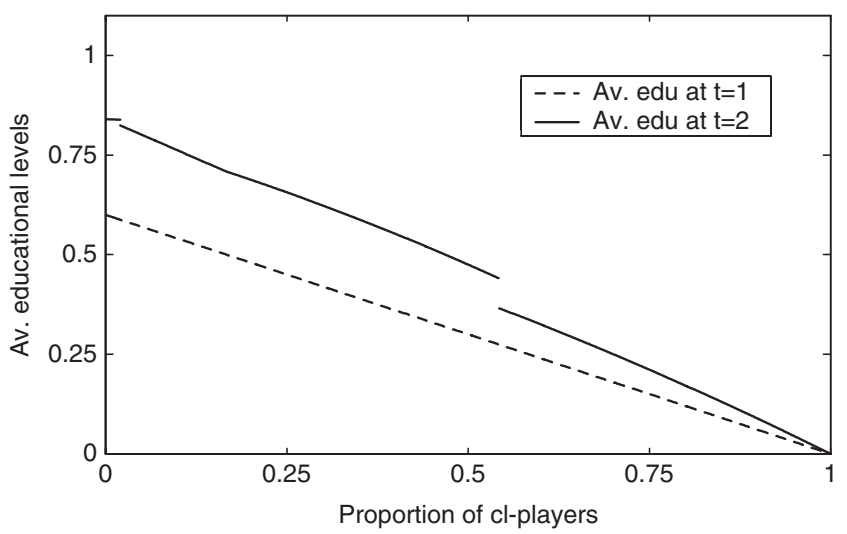

Fig. 5.

Proof The absence of conformist individuals $\left(\alpha_{i l}+\alpha_{i h}+\alpha_{s l}+\alpha_{s h}=1\right)$ implies that $\Delta \bar{e} \geqslant \alpha_{s l} \geqslant 0$. In particular, $\Delta \bar{e}>0$ if $\alpha_{s l}>0$.

\subsection{Conformist, low productivity plus status-seeking, high productivity}

This case captures the situation in which the social preferences of agents are correlated with their innate productivity. Some of the low productivity individuals learn that they cannot emerge and consequently develop a taste for conformism. Some high type individuals realize they have the talent and potential to be above average and thus adopt status-seeking behaviour. Given that $\alpha_{i l}+\alpha_{i h}+\alpha_{c l}+\alpha_{s h}=1$ and $\bar{e}_{1}=\alpha_{i h}+\alpha_{s h}$, the function for $\bar{e}_{2}$ takes the following form:

$$
\bar{e}_{2}=\left\{\begin{array}{l}
\alpha_{i h}+\alpha_{c l}\left(\frac{1}{2} \bar{e}_{1}\right)+\alpha_{s h} \\
\alpha_{i h}+\alpha_{c l}\left(\frac{1}{2} \bar{e}_{1}\right)+\alpha_{s h}\left(2 \bar{e}_{1}\right) \\
\alpha_{i h}+\alpha_{c l}+\alpha_{s h}\left(2 \bar{e}_{1}\right)
\end{array} \quad \Delta \bar{e}= \begin{cases}\alpha_{c l}\left(\frac{1}{2} \bar{e}_{1}\right) & 0.000 \leqslant \bar{e}_{1} \leqslant 0.500 \\
\alpha_{c l}\left(\frac{1}{2} \bar{e}_{1}\right)+\alpha_{s h}\left(2 \bar{e}_{1}-1\right) & 0.500<\bar{e}_{1} \leqslant 0.586 \\
\alpha_{c l}+\alpha_{s h}\left(2 \bar{e}_{1}-1\right) & 0.586<\bar{e}_{1} \leqslant 1.000\end{cases}\right.
$$

Education cannot decrease and increases if $\alpha_{c l}>0$. For low levels of $\bar{e}_{1}$, the growth is driven by individuals of the $c$-class who are trying not to fall behind. The growth in education then becomes faster given that status-seeking individuals, feeling their status in jeopardy, reach higher levels of schooling. Many outcomes are partly separating because $c l$-individuals are indistinguishable from $i h$-individuals for any $\bar{e}_{1}>0.586$.

\subsection{The general case}

The average level of education can both fall or rise when all the six classes of agents are present. It is possible to derive some general results. Let us first consider the uniform distribution defined by $\alpha_{k j}=\frac{1}{6}$ with $k \in\{i, c, s\}$ and $j \in\{l, h\}$ such that $\bar{e}_{1}=0.5$. Optimal choices in $t=2$ are $e_{i l}^{*}=0, e_{i h}^{*}=1, e_{c l}^{*}=0.25, e_{c h}^{*}=1, e_{s l}^{*}=1$ and $e_{s h}^{*}=1$. The resulting average level of education is $\bar{e}_{2}=0.71$ such that $\Delta \bar{e}>0$. Consider next the situation depicted in Fig. 5. In this figure, both $\bar{e}_{1}$ (dashed line) 
and $\bar{e}_{2}$ (solid lines) are expressed as a function of $\alpha_{c l}$ (the proportion of conformist individuals with low productivity) under the assumption that each of the other five categories of workers covers $\frac{1}{5}\left(1-\alpha_{c l}\right)$ of the population. The graph shows that education always increases between the two periods.

Given these two examples, it is easy to predict that, under very weak assumptions regarding the $\alpha_{k j}$, the average level of education will increase over time. In order to have the opposite result, the composition of society has to be strongly biased towards low productivity workers ( $\bar{e}_{1}<0.275$ is a necessary condition) and the majority of highly productive individuals must in addition adopt a conformist behaviour. ${ }^{13}$

\subsection{Summary}

The analysis of the previous subsections highlights the effects that interdependent preferences can have on educational dynamics. In particular, it shows that the presence of individuals who care about their relative position in the educational distribution is a necessary but not sufficient condition for observing an average level of education that evolves over time. Instead, it is a combination of social preferences and productivity levels that triggers these dynamics. The assumptions needed for the model to display a growing level of education are not particularly challenging. For instance, average levels of education are likely to increase even in a society where, in addition to standard agents with independent preferences, there are just a few conformist individuals.

\section{Behaviour of firms}

The previous section focuses on the changes in average levels of education between $t=1$ and $t=2$. These changes are the result of the behaviour of myopic workers who set $E_{1}\left(\tilde{e}_{2}\right)=\tilde{e}_{1}$, i.e., they base their decisions on the assumption that firms use the same hiring policies adopted in $t=1$. In this section, we prove two results. First, we show that if firms actually adopt such a hiring policy, they will probably incur losses. Second, and as a consequence of the first result, we show how sophisticated firms which anticipate correctly the educational trend adjust their screening strategies in order to keep them effective.

Consider one of the main implications of the model, namely that the presence of non-selfish individuals can easily turn perfectly separating outcomes into partly separating ones. In these partly separating outcomes, workers with different productivity acquire the same educational level such that the signals, being no more perfectly correlated with workers' productivity, become less informative. In fact, from the firms' points of view, agents with the same $e^{*}$ are indistinguishable ex ante.

\footnotetext{
${ }^{13}$ As a numerical example consider the case in which $\alpha_{i l}=0.6, \alpha_{i h}=0.05, \alpha_{c l}=0.1, \alpha_{c h}=0.15, \alpha_{s l}=0.05$, $\alpha_{s h}=0.05$. The resulting average education at time $t=1$ is $\bar{e}_{1}=0.25$. Optimal choices at $t=2$ are $e_{i l}^{*}=0$, $e_{i h}^{*}=1, e_{c l}^{*}=0.125, e_{c h}^{*}=0.166, e_{s l}^{*}=1$ and $e_{s h}^{*}=1$ such that $\bar{e}_{2}=0.19$ and $\bar{e}_{2}<\bar{e}_{1}$.
} 
Table 4 consequences on firms' profits in $t=2$

\begin{tabular}{lll}
\hline Interval of $\bar{e}_{1}$ & Classes pooled together & Effects on exp. profits \\
\hline$[0.000,0.275]$ & $i l, c l, c h$ at $e^{*}<1, i h, s l, s h$ at $e^{*}=1$ & mixed \\
$(0.275,0.500]$ & $i h, c h, s l, s h$ at $e^{*}=1$ & negative \\
$(0.500,0.586]$ & $i h, c h, s l$ at $e^{*}=1$ & negative \\
$(0.586,1.000]$ & $i h, c l, c h, s l$ at $e^{*}=1$ & more negative \\
\hline
\end{tabular}

Focusing on firms' expected profits (firms break even when, for each employed worker, the wage matches the productivity), Table 4 shows what would happen in $t=2$ if firms maintain the same screening strategies they used in $t=1$, where they set the discriminatory level at $\tilde{e}_{2}=\tilde{e}_{1}=1$ and offer $w=1$ to any worker with $0 \leqslant e^{*}<1$ and $w=2$ to any worker with $e^{*} \geqslant 1$.

The screening policy such that $\tilde{e}_{2}=\tilde{e}_{1}=1$ may affect profits negatively in the vast majority of cases. The likelihood of these negative effects is weakly increasing with the level of average education. If $\bar{e}_{1} \in[0,0.275]$, firms may either gain or lose; they may hire some conformist individuals with high productivity for the low wage $w=1$ (such that $w_{c h}<\theta_{c h}$ ), but they may also hire some status-seekers with low productivity for the high wage $w=2$ (such that $w_{s l}>\theta_{s l}$ ). However, for any $\bar{e}_{1}>0.275$, firms then run the risk of overpaying workers with a low level of productivity: $w_{s l}>\theta_{s l}$ in the interval $(0.275,0.586]$ and both $w_{c l}>\theta_{c l}$ and $w_{s l}>\theta_{s l}$ in the interval $(0.586,1]$. Such an effect is in line with the initial intuition of the paper. The average level of education is low when just a few individuals have a high level of schooling. These few agents then stand out clearly and send an effective signal to employers. In contrast, the average level of education is high when a considerable fraction of the population has reached high levels of schooling. In this case, signals become less informative and can damage a firm's profitability. Forward-looking firms react to such a trend in accordance with Proposition 5.

Proposition 5 Forward-looking firms correctly anticipate educational trends and optimally adjust their screening policies. In particular, they increase their educational requirements whenever the growth in education is such that signals become noisy and potentially harmful.

Proof Table 4 shows that, with the policy $\tilde{e}_{2}=\tilde{e}_{1}=1$, the probability of overpaying some low productivity workers is weakly increasing in $\bar{e}_{1}$ and strictly positive for any $\bar{e}_{1}>0.275$ (assuming $\alpha_{s l}>0$ ). For example, for any $\bar{e}_{1} \in(0.586,1]$, firms hire and overpay a worker with low productivity with probability $p^{*}=\frac{\alpha_{c}+\alpha_{s l}}{\alpha_{i h}+\alpha_{c l}+\alpha_{c h}+\alpha_{s l}+\alpha_{s h}}>0$. By setting $\tilde{e}_{2} \in\left(1,2 \bar{e}_{1}\right]$ such that $\tilde{e}_{2}>\tilde{e}_{1}$ firms nullify this risk $\left(p^{*}=0\right)$ : in $t=2$ only high productivity individuals (sh-class) get the high wage $w=2$.

The behaviour described by Proposition 5 seems to be consistent with various anecdotal evidence. For example, minimum educational levels required to obtain 
certain jobs have been increasing both in the public and private sector. At the same time, Proposition 5 implies that myopic workers who fail to anticipate firms' behaviours can receive a wage lower than the wage they expected to receive. This is in line with empirical evidence (Brunello et al., 2004, show that European college students substantially overestimate their future wages), as well as with the fact that people often declare themselves to be disappointed with the wages they receive given the level of schooling they acquired.

Moreover, and even if the analysis of a longer game is outside of the scope of this paper, the fact that firms set $\tilde{e}_{2}>\tilde{e}_{1}$ will also have some long-term effects. This higher discriminating level will affect the choices of subsequent cohorts of individuals. Because of economic incentives and social distance considerations, different categories of workers will progressively acquire this new higher level of education which over time will again lose its separating power. According to this argument, workers and firms are involved in a strategic interaction in which both reaction functions are positively sloped such that an escalation in the level of education takes place. A mechanism of this kind is likely to contribute to the rise of average levels of education in Europe.

\section{Conclusion}

The dynamics produced through blending theories of signalling and social distance can help explain the European educational trends of the past few decades. Under very reasonable assumptions about the composition of society, the model endogenously displays an ever-increasing average level of schooling. The presence of status-seeking individuals is not a necessary condition for such growth given that education can grow even in a purely conformist society provided there are enough highly productive individuals. The observation that it is likely, at least for some agents, that the level of schooling may be a way to reach a respectable social position makes the result more robust. The model also rationalizes the fact that as average education increases, signals get progressively less informative and firms become more demanding. The growing number of new postgraduate degrees that are awarded every year provides indirect evidence of the initial intuition. Today a bachelor's degree is a far less effective signal compared to 30 years ago; those who want to stand out need something more.

The social welfare implications of the model are not positive. Asymmetric information about workers' productivity, externalities stemming from interdependent preferences and social pressures not to fall behind in educational distribution suggest a tendency towards the overprovision of education. This indeed seems to be the direction towards which many developed countries are moving.

\section{Acknowledgements}

This work is derived from a chapter in my Ph.D. thesis which was defended in February 2007 at the European University Institute in Florence. I am grateful to the editors and two 
anonymous referees for very helpful suggestions. I also would like to thank Pascal Courty, Andrea Ichino, Karl Schlag, and various seminar participants for useful comments on previous versions of the paper. All remaining errors are mine.

\section{References}

Akerlof, G. (1976) The economics of caste and of the rat race and other woeful tales, Quarterly Journal of Economics, 90, 599-617.

Akerlof, G. (1997) Social distance and social decisions, Econometrica, 65, 1005-27.

Ashenfelter, O., Harmon, C., and Oosterbeek, H. (1999) A review of estimates of the schooling/earnings relationship, with tests for publication bias, Labour Economics, 6, 453-70.

Barro, R.J. (2001) Human capital and growth, American Economic Review, 91, 12-7.

Becker, G. (1993) Human Capital, University of Chicago Press, Chicago.

Bernheim, B.D. (1994) A theory of conformity, The Journal of Political Economy, 102, 841-77.

Brunello, G., Lucifora, C., and Winter-Ebmer, R. (2004) The wage expectations of European business and economics students, Journal of Human Resources, 39, 1116-42.

Cho, I.-K. and Kreps, D.M. (1987) Signaling games and stable equilibria, Quarterly Journal of Economics, 102, 179-221.

Cipollone, P. and Rosolia, A. (2007) Social interactions in high school: lessons from an earthquake, American Economic Review, 97, 948-65.

Dellas, H. and Sakellaris, P. (2003) On the cyclicality of schooling: theory and evidence, Oxford Economic Papers, 55, 148-72.

Duesenberry, J.S. (1949) Income, Saving and the Theory of Consumer Behavior, Harvard University Press, Cambridge, MA.

Eurostat (2001) Educational attainment levels in Europe in the 1990s-some key figures, Statistics in Focus, No. 7.

Eurostat (2005) 17 million tertiary students in the European Union, Statistics in Focus, No. 19.

Falk, A. and Ichino, A. (2006) Clean evidence on peer pressure, Journal of Labor Economics, 24, 29-58.

Fehr, E. and Fischbacher, U. (2002) Why social preferences matter-the impact of nonselfish motives on competition, cooperation and incentives, The Economic Journal, 112, 1-33.

Frank, R.H. (1985) Choosing the Right Pond: Human Behavior and the Quest for Status, Oxford University Press, New York.

Freeman, R. (1971) The Market for College-Trained Manpower, Harvard University Press, Cambridge MA.

Gabaix, X. and Laibson, D. (2006) Shrouded attributes, consumer myopia, and information suppression in competitive markets, Quarterly Journal of Economics, 121, 505-40.

Giannelli, G.C. and Monfardini, C. (2003) Joint decisions on household membership and human capital accumulation of youths - the role of expected earnings and local markets, Journal of Population Economics, 16, 265-85.

Heckman, J., Lochner, L., and Taber, C. (1998) Explaining rising wage inequality: explanations with a dynamic general equilibrium model of labor earnings with heterogeneous agents, Review of Economic Dynamics, 1, 1-58. 
Hopkins, E. and Kornienko, T. (2004) Running to keep in the same place: consumer choice as a game of status, American Economic Review, 94, 1085-107.

Jones, S.R.G. (1984) The Economics of Conformism, Basil Blackwell Publisher, Oxford.

Manski, C. (1993) Adolescent econometricians: how do youth infer the returns to schooling?, in C. Clotfelter and M. Rothschild (eds) Studies of Supply and Demand in Higher Education University of Chicago Press, Chicago.

Manski, C. (2004) Measuring expectations, Econometrica, 72, 1329-76.

Oster, S. and Scott Morton, F. (2005) Behavioral biases meet the market: the case of magazine subscription prices, Advances in Economic Analysis \& Policy, 5, 1-32.

Pollak, R.A. (1976) Interdependent preferences, American Economic Review, 66, 309-20.

Psacharopoulos, G. and Patrinos, H. (2004) Returns to investments in education: a further update, Education Economics, 12, 111-34.

Riley, J. (2001) Silver signals: twenty-five years of screening and signalling, Journal of Economic Literature, 39, 432-78.

Robson, A. (1992) Status, the distribution of wealth, private and social attitudes to risk, Econometrica, 60, 837-57.

Spence, M. (1973) Job market signaling, Quarterly Journal of Economics, 87, 355-74.

Wilson, K., Wolfe, B., and Haveman, R. (2005) The role of expectations in adolescent schooling choices: do youths respond to economic incentives? Economic Inquiry, 43, 467-92.

\section{Appendix}

\section{The case with rational workers}

The main body of the paper studies agents' optimal choices and the dynamics of the average level of education under the assumption of myopic workers and forwardlooking firms. Section 3.2 presents several reasons which support such an assumption. However, for completeness, in this appendix we study the same problem under the more traditional assumption of both workers and firms being forward-looking.

Forward-looking workers base their educational choice on the rational (i.e., ex-post correct) expectation about the future average level of education. More precisely, their utility functions are similar to those presented in Table 2 but $\bar{e}_{t-1}$ is now replaced by $\bar{e}_{t}$ where $\bar{e}_{t}=\sum_{k} \sum_{j} \alpha_{k j} e_{k j}^{t}$ for $k \in\{i, c, s\}$ and $j \in\{l, h\}$. This requires workers to know, or to correctly anticipate, the composition of society (the six parameters $\alpha_{k j}$ ), the behaviour of the various classes of individuals (the six optimal choices $\left.e_{k j}^{t}\right)$ and the critical educational level $\left(\tilde{e}_{t}\right)$ that firms use in order to discriminate among workers with different productivity. ${ }^{14}$

\footnotetext{
${ }^{14}$ Moreover a worker's decision is now potentially strategic because his own choice will influence the average level. We assume the population of workers to be large enough such as to make this effect negligible.
} 
In a perfect Bayesian equilibrium, workers anticipate that firms will design an effective screening scheme. In particular, low productivity individuals realize that firms cannot be deceived and rationally expect to receive the low wage $w=1$. Optimal choices are simply given by the maximization of their utility functions such that $e_{i l}^{*}=0, e_{c l}^{*}=\frac{1}{2} \bar{e}_{t}$ and $e_{s l}^{*}=\frac{2}{3} \bar{e}_{t}$.

Firms anticipate these choices and could therefore set the discriminatory value at $\tilde{e}_{t}=\frac{2}{3} \bar{e}_{t}+\Delta$. So long as $\tilde{e}_{t}$ is smaller than 1 , independent workers with low productivity can profitably deviate from $e_{i l}^{*}=0$ to $e_{i l}^{*}=\tilde{e}_{t}$ (see the basic signalling model in Section 3.1). The screening policy implemented by the firms is thus more elaborate and takes the following form:

$$
\tilde{e}_{t}= \begin{cases}1 & \text { if } \frac{2}{3} \bar{e}_{t}<1 \\ \frac{2}{3} \bar{e}_{t}+\Delta & \text { otherwise }\end{cases}
$$

For highly productive individuals, independent agents choose $e_{i h}^{*}=\tilde{e}_{t}$ (as far as $\tilde{e}_{t}<\sqrt{2}$ ). Conformist individuals have two options: either $e_{c h}^{*}=\frac{2}{3} \bar{e}_{t}$, in which case $w=1$ because they would be pooled with $s$-individuals, or $e_{c h}^{*}=\tilde{e}_{t}$ which leads to $w=2$. Status-seeking individuals also have two options: either $e_{s h}^{*}=\frac{4}{5} \bar{e}_{t}$, which implies $w=1$ if $\frac{4}{5} \bar{e}_{t}<\tilde{e}_{t}$ and $w=2$ otherwise, or $e_{s h}^{*}=2 \bar{e}_{t}$ which leads to $w=1$ if $2 \bar{e}_{t}<\tilde{e}_{t}$ and $w=2$ otherwise.

Assuming that $\alpha_{k j}>0$ for any $k \in\{i, c, s\}$ and any $j \in\{l, h\}$, the only solution that leads to a positive level of average education and is consistent with an optimizing rational behaviour of both the workers and the firms is the following: $e_{i l}^{*}=0, e_{i h}^{*}=1, e_{c l}^{*}=\frac{1}{2} \bar{e}_{t}, e_{c h}^{*}=1, e_{s l}^{*}=\frac{2}{3} \bar{e}_{t}$ and $e_{s h}^{*}=2 \bar{e}_{t}$ with $\tilde{e}_{t}=1$. For any $\bar{e}_{t} \in\left(0, \frac{3}{2}\right)$, this equilibrium is surely perfectly separating. Average education is given by $\bar{e}_{t}=\alpha_{i l}(0)+\alpha_{i h}(1)+\alpha_{c l}\left(\frac{1}{2} \bar{e}_{t}\right)+\alpha_{c h}(1)+\alpha_{s l}\left(\frac{2}{3} \bar{e}_{t}\right)+\alpha_{s h}\left(2 \bar{e}_{t}\right)$ which implies:

$$
\bar{e}_{t}=\frac{\alpha_{i h}+\alpha_{c h}}{1-\frac{1}{2} \alpha_{c l}-\frac{2}{3} \alpha_{s l}-2 \alpha_{s h}}
$$

Rational workers correctly compute the above expression and choose their optimal level of education. As an example, consider the uniform case with $\alpha_{k j}=\frac{1}{6}$ with $k \in\{i, c, s\}$ and $j \in\{l, h\}$. Then $\bar{e}_{t}=\frac{12}{17}$ and $e_{i l}^{*}=0, e_{i h}^{*}=1, e_{c l}^{*}=\frac{6}{17}$, $e_{c h}^{*}=1, e_{s l}^{*}=\frac{8}{17}$ and $e_{s h}^{*}=\frac{24}{17}$. Note that $\bar{e}_{t}>\frac{1}{2}$ where $\frac{1}{2}$ is the average level of education that would have emerged in a standard signalling model where social distance considerations play no role. Therefore social preferences change traditional results even if both firms and workers are forward-looking. But notice also that education remains constant over time: $\bar{e}_{t+1}=\bar{e}_{t}$ for any $t \geqslant 1$. In fact, firms have no reasons to change their screening policy and workers belonging to new cohorts face the same conditions and make the same choices as their predecessors.

To sum up the analysis showed that:

(i) workers' perfect foresight requires individuals to be able to forecast and properly process a large, and possibly prohibitive, amount of information. 
(ii) workers' perfect foresight leads to a static model where the average level of education remains constant over time.

(iii) as a consequence, and in line with the main results of the paper, the assumption of workers' myopia is necessary to rationalize actual educational trends and other related observations (average level of education that changes over time, existence of partly separating outcomes). 